Head trauma

\section{Intracranial injury in minor head trauma}

\section{N Kuppermann}

\section{Commentary on the paper by Dunning et al (see page 653)}

$\mathrm{T}$ raumatic brain injury (TBI) is a leading cause of childhood death and disability worldwide. In the United States, childhood head trauma results in 3000 deaths, 50000 hospitalisations, and 650000 emergency department (ED) visits annually. ${ }^{12}$ Children with seemingly minor head trauma, defined here by Glasgow Coma Scale (GCS) scores of 14-15, account for $40-59 \%$ of those with TBIs, ${ }^{3-5}$ and present a perplexing problem to clinicians. Despite the frequency of childhood minor head trauma, there exists no highly accurate and reliable clinical scoring system for separating those children with minor head trauma at negligible risk of TBI from those at greater risk. Children with TBIs who present with signs of seemingly minor head trauma are at risk for delayed diagnosis and treatment, and such unrecognised TBIs are a source of preventable morbidity. ${ }^{6-8}$ Nevertheless, although some TBIs in children with minor head trauma are initially unrecognised and therefore neuroimaging is delayed, a great many more children evaluated for minor head trauma receive unnecessary neuroimaging.

More than $50 \%$ of children evaluated in EDs for head trauma in the United States undergo cranial computed tomography (CT) scanning, ${ }^{1}$ and this percentage doubled between 1996 and 2000. ${ }^{1}$ Children with minor head trauma account for $70-85 \%$ of children who receive cranial CT scans during their ED evaluation for head trauma in the United States, ${ }^{4}{ }^{5}$ yet only $4-8 \%$ of these CT scans show TBI. ${ }^{1} 5^{9-12}$ Furthermore, approximately $5 \%$ of those with positive CT scans undergo operative intervention. ${ }^{4}$ Therefore CT scans are used inefficiently.

Although CT scanning is the reference standard for diagnosing TBI and delayed recognition of TBI increases morbidity, overuse of CT scanning has important drawbacks. These include transporting the child away from the supervised setting of the ED, the occasional requirement for pharmacological sedation with its potential for complications, and additional health care costs and resource utilisation. The most important drawback of inappropriate CT utilisation, however, is radiation exposure that may lead to malignancy and other sequelae. CT scanning accounts for most $(67 \%)$ of the collective effective radiation dosage from diagnostic imaging in the United States, although only $11 \%$ of all radiographic studies are CT scans. ${ }^{13}$ The typical radiation dosage from a current paediatric cranial CT scan is 300-600 times greater than that for a routine chest radiograph. ${ }^{14}$ The estimates of lifetime attributable risk for fatal cancer from one current generation cranial CT scan ranges from 1 per 2000 scans for young infants to 1 per 5000 for those 10 years old. ${ }^{15}{ }^{16}$ Furthermore, for every case of fatal cancer caused by cranial CT scans, several cases of nonfatal cancer may be induced. ${ }^{15}{ }^{17}$ In addition, it has recently been reported that low doses of ionising radiation in infancy may adversely affect cognitive abilities in young adulthood. ${ }^{18}$ Nevertheless, although the risks of radiation induced malignancy can be estimated, they are small for any given individual.

It is apparent that both under-utilisation and over-utilisation of CT scanning have potential ill effects, and the use of CT scanning following blunt head trauma must be optimised. Unfortunately, there have been few published prospective studies on this topic in children, and none with the necessary size and patient diversity to ensure narrow confidence intervals around the estimates of model accuracy, and wide generalisability of the resulting model. As a consequence, there is substantial variation among ED physicians in the use of CT scans for the evaluation of these children, ${ }^{19-21}$ and the creation of a decision rule to assist with decision making for CT use is a priority among ED physicians. ${ }^{21} 22$

The lack of reliable data on risk factors for TBI in children with minor head trauma motivated Dunning et al to perform a meta-analysis of the literature, which is reported in this issue of Archives. ${ }^{23}$ Using an appropriate, detailed search strategy, the authors identified
16 papers meeting criteria for inclusion in the meta-analysis. In the metaanalysis, the authors assessed the predictive effect of six clinical symptoms and signs, in addition to plain radiography, for identifying which children have intracranial haemorrhage after minor head trauma. The authors reported the pooled estimates for the relative risks of these seven individual criteria. They found that the variables with significant point estimates for risk of TBI were focal neurological findings, skull fracture, a reduced level of consciousness, and a history of loss of consciousness (with seizure trending towards significance).

In the absence of rigorous prospectively acquired data, the meta-analysis by Dunning et al helps us focus on the areas of greatest uncertainty and controversy. There is little debate that a child with focal neurological signs or a depressed level of consciousness after head trauma needs emergent neuroimaging. In addition, several prospective paediatric studies have shown that signs of a skull fracture are significantly predictive of intracranial haemorrhage. ${ }^{45}$ There is much more controversy, however, over the importance of headache, vomiting, and history of loss of consciousness.

Readers must be cautious, however, of translating the findings of Dunning et al into clinical practice. As noted by the authors, the heterogeneous nature of the studies and the wide reported rates of intracranial haemorrhage limit the ability to pool the data and translate the results. Even the definition of "minor head trauma" itself varies between studies. Dunning et al defined minor head trauma as GCS scores of 13-15, whereas others have defined this as GCS scores of $14-15,{ }^{4}$ and GCS scores of $15 .{ }^{9}$ In clinical practice, there is not substantial controversy over imaging children with GCS scores of 13, whereas there is great controversy over children with GCS scores of 15. In our experience, $26.5 \%$ (95\% CI $14.9 \%$ to $41.1 \%$ ) of children with GCS scores of 13 have intracranial haemorrhage versus $2.6 \%$ (95\% CI $1.7 \%$ to $3.9 \%$ ) of those with GCS scores of $15 .^{4}$

Another conundrum is the clinical assessment of pre-verbal children with minor head trauma. As noted by Dunning et al, certain variables cannot be used reliably in the assessment of these children, specifically headache, amnesia, and dizziness. It is unclear how headache was assessed in preverbal children in the studies compiled

Abbreviations: CT, computed tomography; $E D$, emergency department; GCS, Glasgow Coma Scale; TBI, traumatic brain injury 
by Dunning et al. Furthermore, scalp haematoma is one of the most important clinical variables in the assessment of infants with minor head trauma, ${ }^{34}$ and was not assessed in the metaanalysis by Dunning et al. The need for a separate neuroimaging decision rule for pre-verbal children is apparent.

An additional important limitation to the clinical utility of the Dunning et al study is the univariate nature of the meta-analysis. Only by assessing the importance of particular variables in the presence or absence of other variables (through multivariable analysis) can one truly assess the independent predictive effect of any particular variable. Some variables which seem important in univariate analysis may no longer be important in the multivariable analysis. For example, it seems intuitive that a child with a history of loss of consciousness after head trauma has a greater risk of TBI than a child without this history. More controversial, however, is the child with an isolated loss of consciousness (that is, without any other symptoms or signs of TBI). Is this finding in isolation sufficiently predictive of intracranial haemorrhage to require neuroimaging? Several multivariable analyses that assessed this variable, including those in recent large prospective studies, suggest not. ${ }^{4} 591025$

Similarly, other variables which appear not to be predictive in univariate analyses may in fact be predictive when in the presence of other variables. The variables headache and vomiting are not significant in the univariate metaanalysis of Dunning et al, but were important in recent large prospective multivariable studies on this topic. ${ }^{4}$ This may be due to the insufficient characterisation of headache and vomiting in previous retrospective studies on the topic, as noted by Dunning et al. It may also be that these variables emerge as important only in the presence or absence of other variables. Finally, in a meta-analysis such as this, one cannot determine whether the absence of certain high risk factors decreases the overall risk of intracranial haemorrhage below the clinical threshold to obtain emergent neuroimaging.

The Dunning et al study increases our knowledge base about prediction of TBI. It is nonetheless apparent, however, that accurate, sufficient and generalisable data on which to derive reliable evidence based guidelines for neuroimaging children with minor head trauma are still lacking. Fortunately, multicentre research networks in the United Kingdom, Canada, and in the United States have large ongoing studies to gather these data. The Pediatric Emergency Care Applied Research Network (PECARN), a research network of 25 hospital EDs in the United States, ${ }^{26}$ will collect such data on approximately 20000 children with minor head trauma. Using these types of networks to conduct such studies will ensure adequate sample sizes, standardised data collection, appropriate multivariable analyses, and enrolment of a clinical cohort of suitable diversity to generalise the results. These studies will ultimately affect clinical decision making and improve the care of children with minor blunt head trauma.

\section{ACKNOWLEDGEMENTS}

The work and research reflected in this editorial were supported in part by a Distinguished Scholar grant from the United States-United Kingdom Fulbright Commission. I gratefully thank and acknowledge their support.

Arch Dis Child 2004;89:593-594.

doi: 10.1136/adc.2003.046821

Correspondence to: $\operatorname{Dr} \mathrm{N}$ Kuppermann, Division of Emergency Medicine, UC Davis Medical Center, 2315 Stockton Blvd, PSSB Bldg, Suite 2100, Sacramento, CA 98517, USA; nkuppermann@ucdavis.edu

\section{REFERENCES}

1 National Center for Health Statistics Centers for Disease Control and Prevention. 2000; National Hospital Ambulatory Medical Care Survey, Emergency Department File. 2002; CD-ROM Series 13, No. 33.

2 National Center for Injury Prevention and Control. Traumatic brain injury in the United States: Assessing outcomes in children. Centers for Disease Control and Prevention, 2002.

3 Greenes DS, Schutzman SA. Clinical indicators of intracranial injury in head-injured infants. Pediatrics 1999;104:861-7.

4 Palchak MJ, Holmes JF, Vance CW, et al. A decision rule for identifying children at low risk for brain injuries after blunt head trauma. Ann Emerg Med 2003;42:493-506.

5 Quayle KS, Jaffe DM, Kuppermann N, et al. Diagnostic testing for acute head injury in children: when are head computed tomography and skull radiographs indicated? Pediatrics 1997;99:e 1-8.

6 Connors JM, Ruddy RM, McCall J, et al. Delayed diagnosis in pediatric blunt trauma. Pediatr Emerg Care 2001;17:1-4.
7 Furnival RA, Woodward GA, Schunk JE. Delayed diagnosis of injury in pediatric trauma. Pediatrics 1996;98:56-62

8 Klauber MR, Marshall LF, Luerssen TG, et al. Determinants of head injury mortality: importance of the low risk patient. Neurosurgery 1989;24:31-6.

9 Haydel MJ, Shembekar AD. Prediction of intracranial injury in children aged five years and older with loss of consciousness after minor head injury due to nontrivial mechanisms. Ann Emerg Med 2003;42:507-14.

10 Davis RL, Mullen N, Makela M, et al. Cranial computed tomography scans in children after minimal head injury with loss of consciousness. Ann Emerg Med 1994;24:640-5.

11 Homer CJ. American Academy of Pediatrics technical report: blunt head injury in children Pediatrics 1999; 104:e78

12 Schunk JE, Rodgerson JD, Woodward GA. The utility of head computed tomographic scanning in pediatric patients with normal neurologic examination in the emergency department. Pediatr Emerg Care 1996;12:160-5.

13 Mettler FA Jr, Wiest PW, Locken JA, et al. CT scanning: patterns of use and dose. J Radiol Prot 2000:20:353-9.

$14 \mathrm{http} / / /$ cancer.gov/cancerinfo/causes/radiationrisks-pediatric-CT. Accessed 12 February 2004.

15 Brenner DJ. Estimating cancer risks from pediatric $\mathrm{CT}$ : going from the qualitative to the quantitative. Pediatr Radiol 2002;32:228-3.

16 Brenner D, Elliston C, Hall E, et al. Estimated risks of radiation-induced fatal cancer from pediatric CT. AJR 2001;176:289-96.

17 Faulkner K Moores BM. Radiation dose and somatic risk from computed tomography. Acta Radiol 1987; 28:483-8.

18 Hall P, Adami HO, Trichopoulos D, et al. Effect of low doses of ionising radiation in infancy on cognitive function in adulthood: Swedish population based cohort study. BMJ 2004;328: 19 .

19 Aitken ME, Herrerias CT, Davis R, et al. Minor head injury in children: current management practices of pediatricians, emergency physicians, and family physicians. Arch Pediatr Adolesc Med and family physicians.

20 Klassen TP, Reed MH, Stiell IG, et al. Variation in utilization of computed tomography scanning for the investigation of minor head trauma in children: a Canadian experience. Acad Emerg Med 2000;7:739-44

21 Stiell IG, Wells GA, Vandemheen K, et al. Variation in ED use of computed tomography for patients with minor head injury. Ann Emerg Med 1997;30:14-22.

22 Graham ID, Stiell IG, Laupacis A, et al Emergency physicians' attitudes toward and use of clinical decision rules for radiography. Acad Emerg Med 1998;5:134-40.

23 Dunning J, Batchelor J, Stratford-Smith P, et al. A meta-analysis of variables that predict significant intracranial injury in minor head trauma. Arch Dis Child 2004:89:653-9.

24 Greenes DS, Schutzman SA. Clinical significance of scalp abnormalities in asymptomatic headinjured infants. Pediatr Emerg Care 2001;17:88-92.

25 Palchak MJ, Holmes JF, Vance CW, et al. Does an isolated history of loss of consciousness or amnesia predict brain injuries after blunt head trauma? Pediatrics, In press.

26 The Pediatric Emergency Care Applied Research Network. The Pediatric Emergency Care Applied Research Network (PECARN): rationale, development, and first steps. Acad Emerg Med 2003;10:661-8 


\section{The need for caution in considering the diagnostic utility of antibasal ganglia antibodies in movement disorders}

\section{H S Singer, J J Hong, C A Rippel, C A Pardo}

\section{Commentary on the paper by Church et al (see page 611)}

\begin{abstract}
"Now this is not the end. It is not even the beginning of the end. But it is, perhaps, the end of the beginning." (Sir Winston Churchill, Speech in November 1942)
\end{abstract}

n this issue, Church and colleagues discuss the possibility that immunological analyses of sera could serve as diagnostic markers for movement disorders associated with streptococcal infection. ${ }^{1}$ These investigators have been at the forefront of studies evaluating antibasal ganglia antibodies (ABGA) in a variety of proposed poststreptococcal movement conditions. These studies have great potential, given that identification of a specific immune mechanism could lead to insights into pathophysiological mechanisms as well as the development of new therapies. Nevertheless, based on ongoing studies in our laboratory, we feel compelled to raise concerns about the reproducibility of ABGA results, about methodology, and about the inability to confirm serum microinfusion induced alterations of rodent behaviour. As described in the quotation from the wise leader and historian Winston Churchill, we believe that whereas important initial steps have been taken, the final solution has not been reached. The goal of this commentary is not to criticise existing reports, but to identify disparities in data and to emphasise the need for additional testing, before implementing ABGA screening for children with movement disorders.

\section{HYPOTHESIS AND REQUIREMENTS FOR CONFIRMATION}

In susceptible individuals, antibodies produced against the group A $\beta$ haemolytic streptococcus (GABHS) cross-react with epitopes on neurones located in the basal ganglia, through a process of molecular mimicry, and cause movement abnormalities (chorea, tics, other).
IAcceptance of the aforementioned poststreptococcal autoimmune hypothesis requires confirmation on two major levels: (1) epidemiological evidence showing a clear association between streptococcal infection and movement disorders; and (2) definitive evidence for an autoimmune mechanism. Since the focus of this commentary is on laboratory based approaches, the reader is referred to other sources for a discussion of clinical issues and requisites for documentation of a streptococcal infection. $^{2-4}$ Experimental affirmation of autoimmunity requires several factors, including the identification of autoantibodies, the presence of immunoglobulins at the pathological site, the induction of symptoms with autoantigens, passive transfer of the disorder to animal models, and a positive response to immunomodulatory therapy. ${ }^{5}$

\section{METHODS FOR EVALUATING ABGA}

ABGA have been measured in children with SC and TS by either enzyme linked immunosorbent assays (ELISA) or immunofluorescent methods. ELISA is generally selected because it is more specific than immunofluorescent techniques. ${ }^{6}$ Western blot analyses, which permit detection of autoantibody activity against specific brain epitopes, have also been applied successfully. Lastly, the bilateral microinfusion of sera or IgG into rodent striatum has been suggested as a valuable method to assess the functional effect of ABGA obtained from individuals with movement disorders. Although each of the ABGA detection methods appears to be relatively straightforward, results are affected by the use of sera or IgG, brain region (caudate, putamen, or globus pallidus), condition of tissue (fresh or frozen), method of tissue preparation (with or without removal of lipids, that is, delipided), selection of tissue fractions (supernatant, pellet, or synaptosomes), method for Western blotting (electrochemiluminescence, ECL, or colorimetric detection), and several other factors (Singer et al, unpublished data).

\section{NOTEWORTHY LABORATORY DISCREPANCIES}

In their paper, Church and colleagues provide information on antibodies detected in 40 children in the UK presenting with movement disorders associated with streptococcal infections: 20 PANDAS, 16 SC, and four "idiopathic" movement disorders. ${ }^{1}$ Pooled group results suggest that this cohort can be differentiated from a variety of disease controls by ELISA and Western immunoblotting methods. In contrast, results of similar immune assays performed on children with SC and PANDAS and compared to healthy controls, discussed below, have produced significantly different results. ${ }^{7} 8$

\section{ELISA}

In nine children with acute SC, age range 5.8-13.2 years, assays performed on fresh brain tissues from caudate, putamen, and globus pallidus showed that optical density (OD) values were higher in SC patients than controls across all brain regions, but did not reach a level of significance. Although our results $(33 \%$ above a negative cutoff) did not confirm the $95 \%$ positive rates reported by Church and colleagues, ${ }^{6}$ the documented positive trend supports the possibility that inclusion of additional cases would identify a meaningful difference in SC. In contrast, however, two separate evaluations of ELISA in children with PANDAS have shown absolutely no difference from controls $^{8}$ (Singer et al, unpublished data). In the first evaluation, ELISA was measured on supernatant, pellet, and synaptosomal preparations from fresh postmortem caudate, putamen, and globus pallidus by using serum from 15 children with classic PANDAS (participants in the hallmark study by Swedo et $a l^{9}$ ). This lack of correlation has been further confirmed in a second study of 40 additional cases of PANDAS. Thus, ELISA is unchanged in a population of children who have a proposed association with GABHS. Nevertheless, since ABGA are identified in normal individuals, it is possible that small changes could be missed or the serum might be obtained after symptoms have waned.

Abbreviations: $A B G A$, antibasal ganglia antibodies; GABHS, group $A \beta$ haemolytic streptococcus; PANDAS, paediatric autoimmune neuropsychiatric disorders associated with streptococcal infections; SC Sydenham's chorea; TS, Tourette syndrome 


\section{Western immunoblotting}

In this technique, small amounts of brain protein are subjected to electrophoresis in acrylamide gels, the protein is transferred to nitrocellulose, exposed to serum, and then to an antihuman IgG secondary antibody that is conjugated to either horseradish peroxidase or a colorimetric agent. Resulting bands are then analysed by several techniques ranging from direct visual comparison (useful with minimal bands) to digital conversion of bands on each blot to peaks with assigned molecular weights and measurable peak heights and areas.

Church and colleagues, using frozen tissue and a colorimetric assay, identified only a few bands in controls (limited reactivity against any basal ganglia antigens), but significantly more bands in poststreptococcal patients. ${ }^{1}$ They suggest that specific MW bands $(60,45,40 \mathrm{kDa})$ are commonly detected in their movement disorder patients but not in controls. Their identification of similar bands in subjects with different clinical disorders raises questions of disease specificity. In addition, as shown in fig 1 , the use of fresh postmortem tissue and an ECL detection system identifies many more bands than shown by the method used by Church and colleagues (frozen tissue and a colorometric detection system). This suggests that the methods selected can have an important influence on the detection of potential disease specific basal ganglia antigens. A further issue is that antigen binding, claimed to be associated with PANDAS and SC patients, is also found in controls. That is, in the evaluation of fresh postmortem tissue without lipids from 10 control subjects, binding was present at $60 \mathrm{kDa}$ in $100 \%$, at $45 \mathrm{kDa}$ in $80 \%$, and at $40 \mathrm{kDa}$ in $100 \%$; binding in frozen delipided tissue was present in $100 \%, 30 \%$, and $40 \%$, respectively (Singer et al, unpublished data). Lastly, questions exist about the importance of

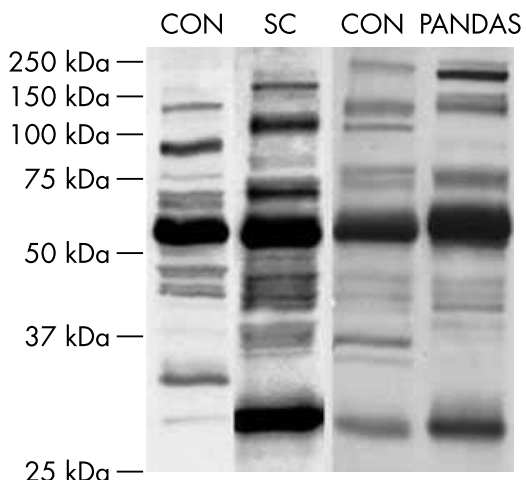

Figure 1 Western blotting using sera and caudate supernatant. Numerous bands (antigen-antibody interactions) are observed in SC and PANDAS subjects and controls. a band at $60 \mathrm{kDa}$, since epitopes at this same molecular weight are identified using only secondary antibody, without the addition of serum or IgG (fig 2).

Western immunoblotting of serum from patients with SC, analysed by use of discriminant analysis of data vectors calculated from individual blots, has shown that patterns are significantly different from controls, most notably in caudate supernatant fractions. Numerous antigens contributed to this statistical difference, but the two most prominent molecular masses that distinguished the groups were at 126 and $113 \mathrm{kDa}^{7}$ In PANDAS, complex staining patterns were observed in all cases, but no differences from control were seen for either the number of bands or the total density of bands. Discriminant analyses did show small differences in mean data vectors between PANDAS and controls that were limited to the caudate supernatant preparation. These changes, however, were minimal compared to those detected between SC and controls. Whether differences in Western blotting offer specific insight into the pathophysiology of movement disorders or merely represent an epiphenomenon, remains undetermined. sis of TS and no history of streptococcal associated exacerbations show prominent immunoblot differences. ${ }^{10}$ To date, weaknesses in most ABGA studies include the analysis of antibody patterns at only a single point in time, rather than in longitudinal samples, and the failure to determine whether there is an association between ABGA and a For example, children with the diagno-

streptococcal infection. Lastly, it should be recognised that measured autoantibody repertoires can be secondary immune responses to components of damaged tissue ${ }^{11}$ rather than to the inciting pathological factor.

\section{Indirect immunofluorescence}

Studies in the Church and Singer laboratories have identified fluorescent binding in some patients with TS and PANDAS. Location of this positive binding remains unclear, ranging from axonal with sparing of the cell body to astroglial processes. Additional analyses with double labelled staining (antihuman IgG with GFAP or a neuronal indicator) are necessary to further identify the precise anatomical region. Similar positive binding has also been detected in controls.

\section{RODENT MICROINFUSION MODELS}

One of the major criteria for any neurological disorder to be recognised as autoimmune is the ability to transfer the disease passively to animals. Two studies, both frequently cited as supporting evidence for an autoimmune mechanism in paediatric movement disorders, have reported behavioural effects after the infusion of ABGA from subjects with TS into rodent striatum. ${ }^{12}{ }^{13}$ That is, the microinfusion of sera containing increased antibodies into the ventral or ventrolateral striatum of rats significantly increases stereotypic behaviours (for example, licks and forepaw shakes) and in one study caused episodic utterances. ${ }^{12}$ In contrast to the

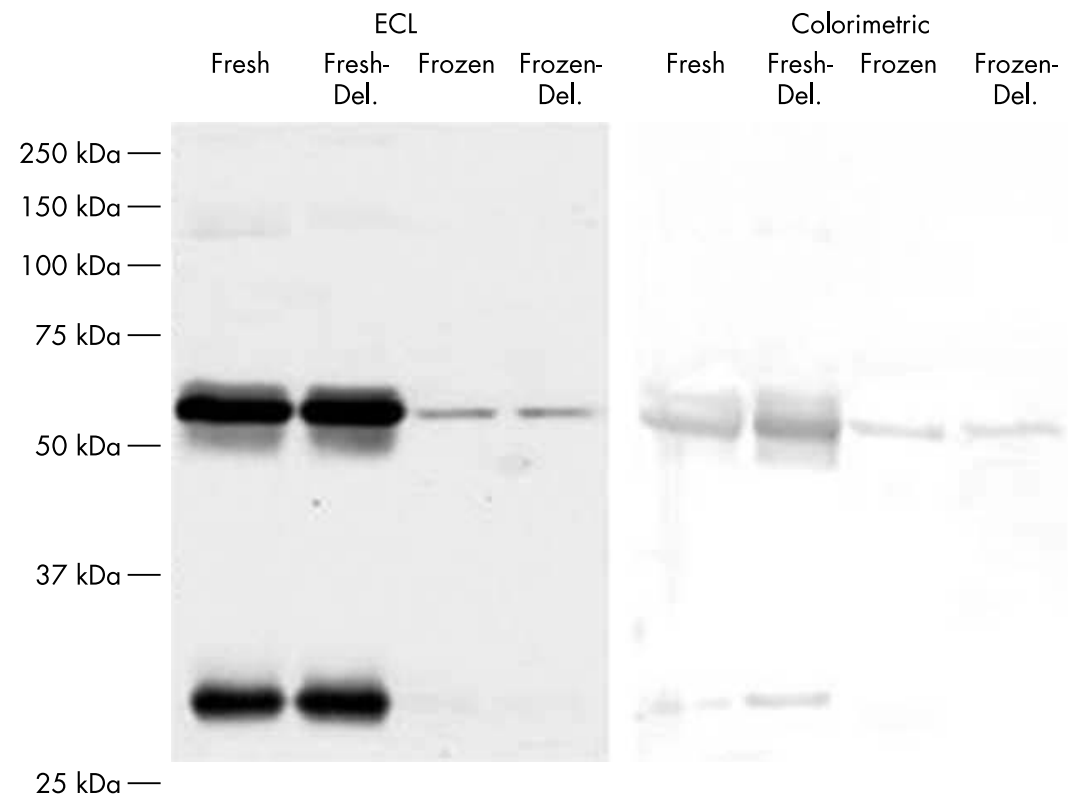

Figure 2 Two Western blotting methods using various caudate preparations and only secondary antibody. Despite the lack of serum (source of the primary antibody), bands are seen at 60 and $28 \mathrm{kDa}$. Del., delipided. 
aforementioned, however, two additional studies have questioned the validity of the model. For example, microinfusion of sera from patients with TS and PANDAS into the same striatal sites as in prior reports, failed to induce any significant increase in either stereotypic behaviour or episodic utterances. ${ }^{14}$ Lastly, a double blind, collaborative effort involving three institutions, showed no differences in behaviours between infusions of TS sera with high and low ABGA titres, but non-specific increases induced by continuous infusion (Singer et al, unpublished data).

\section{CONCLUSION}

In conclusion, although it is currently hypothesised that an autoimmune process is the underlying pathophysiological mechanism in several movement disorders, specific disease related brain autoantigens have not been identified. Furthermore, before accepting the pathogenic relevance of a monoclonal antibody, confirmation will require determination of regional and neuronal specificity, evidence of cross reactivity with streptococcal proteins, and documentation of passive transfer of the disorder. We believe that the proposal of an autoimmune ABGA mechanism for poststreptococcal neurological disorders deserves careful study, but emphasise that the use of ABGA as diagnostic markers is currently premature.

For clinicians considering making the diagnosis of PANDAS, we suggest strict adherence to the formal published criteria, ${ }^{10}$ recognition that the diagnosis requires longitudinal assessments, realisation that a single measurement of antistreptococcal antibodies has limited value, and that treatment with prophylactic antibiotics or immunomodulatory therapies is controversial and fraught with potential serious side effects. Until further clarification is available, we suggest that therapy should continue to focus on the use of standard approaches to control symptoms. Along with the scientific community, we anxiously await the result of longitudinal case-control studies now in progress.

\section{ACKNOWLEDGEMENTS}

Supported in part by funding from NIH ROI NS37706, RO1 MH61940, and ROI NS42240.

\section{Arch Dis Child 2004;89:595-597.}

doi: 10.1136/adc.2003.046847

\section{Authors' affiliations}

H S Singer, J J Hong, C A Rippel, C A Pardo, Department of Neurology, Johns Hopkins University School of Medicine, Jefferson Street Building 124, 600 N Wolfe Street, Baltimore, MD, USA

Correspondence to: $\mathrm{Dr} \mathrm{H}$ S Singer, Division of Pediatric Neurology, Johns Hopkins Hospital, Jefferson Street Building 124, $600 \mathrm{~N}$ Wolfe Street, Baltimore, MD 21287-1000, USA; hsinger@jhmi.edu

\section{REFERENCES}

1 Church AJ, Dale RC, Giovannoni G. Anti-basal ganglia antibodies: a possible diagnostic utility in idiopathic movement disorders? Arch Dis Child 2004;89:611-4.

2 Singer HS, Loiselle C. PANDAS: a commentary. J Psychosom Res 2003;55:31-9.

3 Kurlan R. Tourette's syndrome and 'PANDAS': will the relation bear out? Pediatric autoimmune neuropsychiatric disorders associated with streptococcal infection. Neurology 1998:50:1530-4.

4 Kaplan EL, Gerber MA. Group A, group C, and group $G$ beta hemolytic streptococcal infections. In: Feigin RD, Cherry JD, eds. Textbook of pediatric infectious diseases, Vol. 1. Philadelphia: W. B Saunders, 1998:1076-88.

5 Archelos JJ, Hartung HP. Pathogenetic role of autoantibodies in neurological diseases. Trends Neurosci 2000;23:317-27.

6 Church AJ, Cardoso F, Dale RC, et al. Anti-basal ganglia antibodies in acute and persistent Sydenham's chorea. Neurology 2002;59:227-31

7 Singer HS, Loiselle CR, Lee O, et al. Antibasal ganglia antibody abnormalities in Sydenham chorea. J Neuroimmunol 2003;136:154-61.

8 Singer HS, Loiselle CR, Lee O, et al. Anti-basal ganglia antibody abnormalities in PANDAS. Mov Disord 2004:19:406-15.

9 Swedo SE, Leonard HL, Garvey M, et al. Pediatric autoimmune neuropsychiatric disorders associated with streptococcal infections: clinical description of the first 50 cases. Am J Psychiatry 1998; 155:264-71.

10 Singer HS, Giuliano JD, Hansen BH, et al. Antibodies against human putamen in children with Tourette syndrome. Neurology 1998:50:1618-24.

11 Bornstein NM, Aronovich B, Korczyn AD, et al. Antibodies to brain antigens following stroke. Neurology 2001;56:529-30.

12 Hallett JJ, Harling-Berg CJ, Knopf PM, et al. Antistriatal antibodies in Tourette syndrome cause neuronal dysfunction. I Neuroimmunol 2000;111:195-202.

13 Taylor JR, Morshed SA, Parveen S, et al. An animal model of Tourette's syndrome. Am J Psychiatry 2002; 159:657-60.

14 Loiselle CR, Lee O, Moran TH, et al. Striata microinfusion of Tourette syndrome and PANDAS sera: failure to induce behavioral changes. Mov Disord 2004; 19:390-6.

\section{IMAGES IN PAEDIATRICS}

\section{A novel cause of neck stiffness}

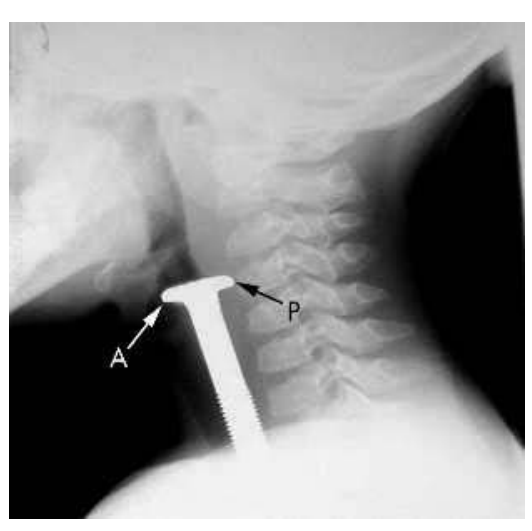

A 4 year old boy was referred to hospital with suspected meningitis. There was a four day history of neck stiffness and anorexia but also of drooling and dysphonia. Examination revealed him to be significantly dehydrated but alert and afebrile. There was no stridor. Serum urea and electrolytes were consistent with hypernatraemic dehydration: sodium $161 \mathrm{mM} / \mathrm{l}$; urea $16.9 \mathrm{mM} / \mathrm{l}$; creatinine $74 \mu \mathrm{M} / \mathrm{l}$. A lateral radiograph of the neck showed a large foreign body in the proximal oesophagus at the level of cricopharyngeus (see fig). After appropriate fluid resuscitation, the foreign body was removed under general anaesthesia revealing a significant ulceration in the posterior superior pharynx and minor oedema of the arytenoids corresponding with $\mathrm{P}$ and $\mathrm{A}$ on the figure respectively. The child's parents identified the foreign body as a bolt recently noted to be absent from its hole in a bed-head. The postoperative recovery was swift and complete. The discharge weight was almost $15 \%$ greater than the weight on presentation. This case illustrates how cricopharyngeus ("the upper oesophageal sphincter") is one of the three sites where oesophageal foreign bodies may lodgethe other two sites being where the aortic arch descends anterior to the mid oesophagus and at the level of the cardiac sphincter. The case also serves as a reminder that young children can conceal a potential life threatening condition for several days.

R Gupta, C Driver, $\mathbf{S}$ Turner Department of Child Health, University of Aberdeen, Foresterhill, Aberdeen AB25 2ZG, UK; s.w.turner@abdn.ac.uk 